\title{
COARSE DYNAMICS AND FIXED-POINT THEOREM
}

\author{
TOMOHIRO FUKAYA
}

\begin{abstract}
We study semigroup actions on a coarse space and the induced actions on the Higson corona from a dynamical point of view. Our main theorem states that if an action of an abelian semigroup on a proper coarse space satisfies certain conditions, the induced action has a fixed point in the Higson corona. As a corollary, we deduce a coarse version of Brouwer's fixed-point theorem.
\end{abstract}

\section{$\S 1$. Introduction}

A metric space $X$ is proper if closed, bounded sets in $X$ are compact. Let $X$ and $Y$ be proper metric spaces, and let $f: X \rightarrow Y$ be a map (not necessarily continuous). We define the following.

(a) The map $f$ is proper if for each bounded subset $B$ of $Y, f^{-1}(B)$ is a bounded subset of $X$.

(b) The map $f$ is bornologous if for every $R>0$ there exists $S>0$ such that for each $x, y \in X, d(x, y)<R$ implies that $d(f(x), f(y))<S$.

(c) The map $f$ is coarse if it is proper and bornologous.

Let $f, g: X \rightarrow Y$ be maps. We define $f$ as close to $g$, denoted $f \simeq g$, if there exists $R>0$ such that $d(f(x), g(x))<R$ for all $x \in X$. We define $X$ and $Y$ as coarsely equivalent if there exist coarse maps $f: X \rightarrow Y$ and $g: Y \rightarrow X$ such that $g \circ f$ and $f \circ g$ are close to the identity maps of $X$ and $Y$, respectively. A coarse space is a coarsely equivalent class of proper metric spaces. The category of coarse spaces consists of coarse spaces and coarse maps.

Let $\varphi: X \rightarrow \mathbb{C}$ be a bounded continuous map. For each $r>0$, we define a map $V_{r} \varphi: X \rightarrow \mathbb{R}$ by

$$
V_{r} \varphi(x):=\sup \{|\varphi(y)-\varphi(x)|: d(x, y)<r\}
$$

Received December 10, 2009. Revised May 12, 2010. Accepted May 14, 2010.

2010 Mathematics Subject Classification. Primary 55C20; Secondary 20 F65.

Author's work supported in part by the Japan Society for the Promotion of Science grant (19.3177).

(C) 2011 by The Editorial Board of the Nagoya Mathematical Journal 
We define $\varphi$ as a Higson function if for each $r>0, V_{r} \varphi$ vanishes at infinity. The Higson functions on a proper metric space $X$ form a unital $C^{*}$-algebra, denoted by $C_{h}(X)$. It follows from the Gelfand-Naimark theorem that there exists a compactification $h X$ of $X$ such that $C(h X)=C_{h}(X)$. The compactification $h X$ is called the Higson compactification. Its boundary $h X \backslash X$ is denoted by $\nu X$ and called the Higson corona. The Higson corona is a functor from the category of coarse spaces into the category of compact Hausdorff spaces. Namely, a coarse map $f: X \rightarrow Y$ induces a unique continuous map $\nu f: \nu X \rightarrow \nu Y$, and moreover, if coarse maps $f, g: X \rightarrow Y$ are close, then $\nu f=\nu g$. It follows that the Higson coronae of two proper metric spaces which are coarsely equivalent to each other are homeomorphic. We note that the Higson corona of an unbounded proper metric space is never second countable and that its cardinality is greater than or equal to $2^{2^{\aleph_{0}}}=2^{\mathfrak{c}}$. This means that the Higson corona is a huge, extremely complicated space. We refer to [5] for a general reference of coarse geometry and the Higson compactification.

Let $X$ be a proper metric space, and let $G$ be a finitely generated semigroup acting on $X$. Here $G$ is a proper metric space with a left-invariant word metric. A coarse action, defined below, of $G$ on $X$ induces a continuous action of $G$ on the Higson corona $\nu X$. The main subject of this article is to study fixed-point properties of these actions.

Definition 1.1. An action of $G$ on $X$ is a coarse action if for each element $g$ of $G$, the map $\Psi_{g}: X \rightarrow X$ defined by $x \mapsto g \cdot x$ is a coarse map.

Definition 1.2. For a point $x_{0}$ of $X$, the orbit map $\Phi_{x_{0}}: G \rightarrow X$ is defined by $g \mapsto g \cdot x_{0}$. We define the following.

(a) The orbit of $x_{0}$ is proper if $\Phi_{x_{0}}$ is proper.

(b) The orbit of $x_{0}$ is bornologous if $\Phi_{x_{0}}$ is bornologous.

(c) The orbit of $x_{0}$ is coarse if $\Phi_{x_{0}}$ is coarse.

The action of $G$ on itself is a typical example of the coarse action (see Lemma 2.1).

TheOREM 1.3. Assume that $G=\mathbb{N}^{k}$ or $\mathbb{Z}^{k}$ and that $G$ acts on $X$ as a coarse action. Suppose that there exists a point $x_{0}$ of $X$ whose orbit is coarse. Then the induced action of $G$ on the Higson corona $\nu X$ has a fixed point. Namely, there exists a point $x$ of $\nu X$ such that $g \cdot x=x$ for any element $g \in G$. 
Moreover, let $\bar{X}$ be a coarse compactification of $X$ (see Definition 2.2). We suppose that the action of $G$ on $X$ is continuous and extends to a continuous action on $\bar{X}$. Then the action on the boundary $\partial X=\bar{X} \backslash X$ has a fixed point. Namely, there exists a point $z$ of $\partial X$ such that $g \cdot z=z$ for any element $g \in G$.

EXAMPLE 1.4. Let $G$ be a finitely generated group with an element $h$ of infinite order. Then a group action of $\mathbb{Z}$ on $G$ by $(n, g) \mapsto h^{n} g$ is a coarse action, and the orbit of $e \in G$ is coarse. Thus, the action of $\mathbb{Z}$ on the Higson corona $\nu G$ has a fixed point. Moreover, if $G$ is a hyperbolic group, this action extends to the Gromov boundary $\partial_{g} G$ (see Proposition 2.4 and Example 2.5). Then this action of $\mathbb{Z}$ on $\partial_{g} G$ has a fixed point. This is a well-known fact on the boundary of hyperbolic groups (see [2, Chapter 8, Proposition 10 and Theorem 30]).

EXAMPLE 1.5. The wreath product $\mathbb{Z} \imath \mathbb{Z}$ contains $\mathbb{Z}^{n}$ as a subgroup for any positive integer $n$ (see $[5$, p. 135$]$ ). Thus, the action of $\mathbb{Z}^{n}$ on $\mathbb{Z} \imath \mathbb{Z}$ is coarse, and the induced action of $\mathbb{Z}^{n}$ on $\nu(\mathbb{Z} \imath \mathbb{Z})$ has a fixed point.

REMARK 1.6. Let $F_{2}$ be a free group of rank 2. By Lemma 2.1, the action of $F_{2}$ on $F_{2}$ is a coarse action and has a coarse orbit. However, the induced action of $F_{2}$ on the Higson corona $\nu F_{2}$ does not have any fixed point (see Proposition 2.8).

Let $f: X \rightarrow X$ be a coarse map. We call a point $x$ of $X$ a coarse fixed point of $f$ if the orbit of $x,\left\{f^{n}(x): n \in \mathbb{N}\right\}$, is bounded.

Corollary 1.7 (coarse version of Brouwer's fixed-point theorem). Let $X$ be a proper metric space. Suppose that $f: X \rightarrow X$ is an isometry and that $\bar{X}$ is a coarse compactification of $X$ such that $f$ extends to a continuous map $\bar{f}: \bar{X} \rightarrow \bar{X}$. If $f$ has no coarse fixed point in $X$, then $\bar{f}$ has a fixed point in $\partial X$.

In this paper, isometries does not mean surjective isometries. This corollary says that an isometry $f$ always has a coarse fixed point in $X$ or a fixed point in $\partial X$.

EXAMPLE 1.8. The Gromov boundary of the hyperbolic plane $\mathbb{H}^{2}$ is $S^{1}$. Let $f: \mathbb{H}^{2} \rightarrow \mathbb{H}^{2}$ be a continuous map such that $f$ extends to the Gromov boundary. Then Brouwer's fixed-point theorem says that $f: \mathbb{H}^{2} \cup S^{1} \rightarrow \mathbb{H}^{2} \cup$ $S^{1}$ has a fixed point. Let $\Gamma$ be a discrete group of isometries acting freely on $\mathbb{H}^{2}$ with quotient a compact surface. Here $\Gamma$ is coarsely equivalent to $\mathbb{H}^{2}$, 
and its Gromov boundary is also $S^{1}$. Let $f: \Gamma \rightarrow \Gamma$ be an isometry. Then Corollary 1.7 says that $f: \Gamma \cup S^{1} \rightarrow \Gamma \cup S^{1}$ has a coarse fixed point in $\Gamma$, or a fixed point in $S^{1}$.

EXAmPLE 1.9. Let $M$ be a compact path metric space. The cone $C M$ on $M$ is the quotient space $M \times[0, \infty) / \sim$, where $(x, t) \sim\left(x^{\prime}, t^{\prime}\right)$ if and only if either $x=x^{\prime}$ and $t=t^{\prime}$ or $t=t^{\prime}=0$. We define a continuous function $\tau:[0, \infty) \rightarrow[0,1)$ by

$$
\tau(t)=\frac{t}{1+t}
$$

Set $\overline{C M}=M \times[0,1] / \sim$. The embedding $C M \rightarrow \overline{C M}:(x, t) \mapsto(x, \tau(t))$ gives us a compactification of $C M$. We can define an appropriate metric on $C M$ such that $C M$ is a proper metric space. Then $\overline{C M}$ becomes a coarse compactification (see Appendix). Let $f: C M \rightarrow C M$ be an isometry, and we suppose that $f$ extends to a continuous map $\bar{f}: \overline{C M} \rightarrow \overline{C M}$. Then $f$ has a coarse fixed point in $C M$, or $\bar{f}$ has a fixed point on the boundary $\partial(C M) \cong M$. If $M$ is Euclidean neighborhood retract (ENR), then the Lefschetz fixed-point theorem implies that $\bar{f}$ has a fixed point since $\overline{C M}$ is contractible. However, let $g$ be an isometry on a punctured cone $C M^{\times}=M \times[1 / 2, \infty)$. We suppose that $g$ extends to a continuous map $\bar{g}$ on a coarse compactification $\overline{C M^{\times}}=M \times[1 / 2,1]$. Then $g$ also has a coarse fixed point in $C M^{\times}$, or $\bar{g}$ has a fixed point in the boundary $\partial(C M) \cong M$. Since $C M^{\times}$is homotopic to $M$, the Lefschetz fixed-point theorem does not imply this.

ExAmple 1.10. Set $M=S^{n-1}$. Then $C M$ is homeomorphic to the $n$ dimensional Euclidean space $\mathbb{R}^{n}$, and $\overline{C M}$ is homeomorphic to unit ball $B^{n}=\left\{x \in \mathbb{R}^{n}:|x| \leq 1\right\}$. Any isometry $f: \mathbb{R}^{n} \rightarrow \mathbb{R}^{n}$ which can be extended to continuous map $\bar{f}: B^{n} \rightarrow B^{n}$ has a coarse fixed point in $\mathbb{R}^{n}$ or a fixed point in $\partial\left(\mathbb{R}^{n}\right)=S^{n-1}$.

REMARK 1.11. In Corollary 1.7, the assumption that the map $f$ is an isometry is essential. See Section 4.

\section{$\S 2$. Coarse action}

Lemma 2.1. Let $G$ be a finitely generated group or $G=\mathbb{N}^{k}$ with leftinvariant word metric for some generating set. The action of $G$ on $G$ given by the left-translation $(g, h) \mapsto g h$ is a coarse action. Furthermore, any orbit of $h \in G$ has a coarse orbit. 
Proof. For given $g \in G$, the map $\Psi_{g}: G \rightarrow G$ given by $h \mapsto g h$ is an isometry, so it is a coarse map. Let $h \in G$ be given; we consider the orbit map $\Phi_{h}: G \rightarrow G$ given by $g \mapsto g h$. We denote the word length by $|\cdot|$. For any $g, g^{\prime} \in G$, we have

$$
d\left(\Phi_{h}(g), \Phi_{h}\left(g^{\prime}\right)\right)=d\left(g h, g^{\prime} h\right)=\left|h^{-1} g^{-1} g^{\prime} h\right| \leq\left|g^{-1} g^{\prime}\right|+2|h| .
$$

This shows that $\Phi_{h}$ is a large-scale Lipschitz map and hence a bornologous map. Let $D$ be a bounded subset of $G$, which is a finite set. Since $\Phi_{g}$ is injective, $\sharp \Phi_{g}^{-1}(D) \leq \sharp D<\infty$. This shows that $\Phi_{h}$ is a proper map. It follows that $\Phi_{h}$ is a coarse map and that the orbit of $h$ is coarse.

The coarse action of $G$ on $X$ induces the continuous action on the Higson corona of $X$. Since the Higson corona is an extremely complicated space, it is useful to find more manageable compactifications. The following show the relation between the Higson corona and such compactifications. We say that $E \subset X \times X$ is a controlled set if there exists $R>0$ such that any $(x, y) \in E$ satisfies $d(x, y)<R$ (see [5, Chapter 2]).

Definition 2.2. Let $X$ be a proper metric space, and let $\bar{X}$ be a compactification of $X$. We call $\bar{X}$ a coarse compactification if the following condition is satisfied: for any controlled set $E \subset X \times X$ of $X$, its closure $\bar{E}$ in $\bar{X} \times \bar{X}$ meets the boundary $\partial(X \times X)=\bar{X} \times \bar{X} \backslash(X \times X)$ only at the diagonal; that is,

$$
\bar{E} \cap \partial(X \times X) \subset \Delta_{\partial X}=\{(\omega, \omega): \omega \in \partial X\} .
$$

Proposition 2.3. The Higson compactification $h X$ is a coarse compactification. Moreover, it is a universal coarse compactification, in the sense that, for any coarse compactification $\bar{X}$ of $X$, the identity map id: $X \rightarrow X$ extends uniquely to a continuous surjective map $\iota: h X \rightarrow \bar{X}$.

Proposition 2.4. Let $f: X \rightarrow X$ be a continuous coarse map, and let $\bar{X}$ be a coarse compactification. Suppose that $f$ extends to a continuous map $\bar{f}: \bar{X} \rightarrow \bar{X}$. Then $\bar{f}(\partial X) \subset \partial X$ and the following diagram commutes:

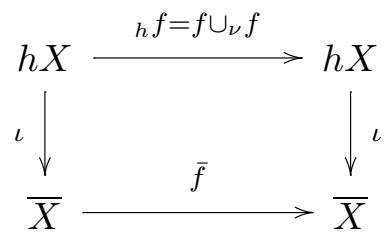


Proof. By the properness of $f$, we have $\bar{f}(\partial X) \subset \partial X$. Since $f$ is a continuous coarse map, $f$ induces $f^{*}: C_{h}(X) \rightarrow C_{h}(X)$. Because $f$ extends to a continuous map on $\bar{X}, f^{*}$ maps $C(\bar{X})$ to itself. It is clear that $f^{*}$ and the inclusion $\iota^{*}: C(\bar{X}) \hookrightarrow C_{h}(X)$ commute.

ExAmple 2.5. Let $X$ be a Gromov hyperbolic space. Then the Gromov compactification of $X$ is a coarse compactification (see [5, Lemma 6.23]). If $G$ is a hyperbolic group and $\gamma \in G$ has an infinite order, then the lefttranslation $\gamma \cdot G \rightarrow G$ extends to a continuous map on the Gromov boundary $\partial_{g} G$ (see $[2$, Chapters 7,8$]$ ).

Lemma 2.6. Let $G$ be $\mathbb{N}^{k}$ or $\mathbb{Z}^{k}$. The coarse action of $G$ on $G$ by $(g, n) \mapsto$ $g+n$ extends to the trivial action on the Higson corona of $G$. Namely, for any $g \in G$, the induced $\operatorname{map}_{\nu} \Psi_{g}: \nu G \rightarrow \nu G$ is equal to the identity map.

Proof. Let $g \in G$ be given. For any $n \in G, d\left(n, \Psi_{g}(n)\right)=d(n, g+n)=|g|$. Then $\Psi_{g}$ is close to the identity $\operatorname{id}_{G}: G \rightarrow G$. It follows that ${ }_{\nu} \Psi_{g}=\operatorname{id}_{\nu G}$.

Proof of Theorem 1.3. Let $x_{0}$ be a point in $X$ whose orbit is coarse. The $G$-equivariant map $\Phi_{x_{0}}: G \rightarrow X$ given by $g \mapsto g \cdot x_{0}$ is a coarse map. Thus, $\Phi_{x_{0}}$ extends to the continuous map ${ }_{\nu} \Phi_{x_{0}}: \nu G \rightarrow \nu X$. For each $g \in G$, the coarse map $\Psi_{g}: X \rightarrow X$ extends to the continuous map ${ }_{\nu} \Psi_{g}: \nu X \rightarrow \nu X$. Then we have the following commutative diagram:

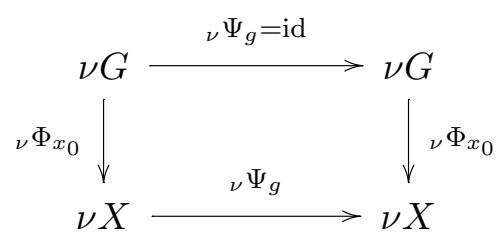

By Lemma 2.6, the upper right arrow is the identity map. We choose any $x^{\prime} \in \nu G$, and we put $x={ }_{\nu} \Phi_{x_{0}}\left(x^{\prime}\right)$. Then by the above commutative diagram, we have ${ }_{\nu} \Psi_{g}(x)=x$.

Moreover, let $\bar{X}$ be a coarse compactification of $X$. We suppose that the action of $G$ on $X$ is continuous and extends to a continuous action on $\bar{X}$. Then we have the following commutative diagram:

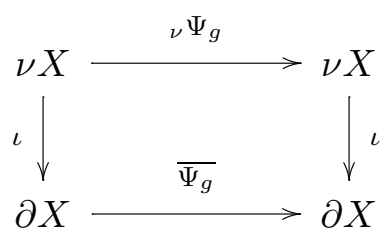


Set $z=\iota(x)$. We have $\bar{\Psi}_{g}(z)=z$.

REMARK 2.7. Suppose that $X$ is a proper metric space and that $\Phi_{x_{0}}$ is a continuous map. By the results of Dranishnikov, Keesling, and Uspenskij [1], if $\Phi_{x_{0}}$ is a coarse embedding and $\Phi_{x_{0}}(G)$ is a closed subset, we can show that the map ${ }_{\nu} \Phi_{x_{0}}$ in the proof of Theorem 1.3 is an embedding. Indeed, [1, Theorem 1.4] states that the closure of $\Phi_{x_{0}}(G)$ in $h X$ is homeomorphic to the Higson corona $\nu\left(\Phi_{x_{0}}(G)\right)$ of $\Phi_{x_{0}}(G)$, which is a metric space with the induced metric from $X$. Since $\Phi_{x_{0}}$ is a coarse embedding, $\nu G$ is homeomorphic to $\nu\left(\Phi_{x_{0}}(G)\right)$. Thus, ${ }_{\nu} \Phi_{x_{0}}: \nu G \rightarrow \nu X$ is an embedding. It follows that there are $2^{2^{\aleph_{0}}}$ fixed points on the Higson corona $\nu X$.

We cannot generalize Theorem 1.3 to a free group action, as is shown by the following.

Proposition 2.8. The action of $F_{2}$ on $\nu F_{2}$ induced by the left-translation $F_{2} \times F_{2} \rightarrow F_{2}$ has no fixed point. Namely, there exists no point $x$ of $\nu F_{2}$ such that $g \cdot x=x$ for any element $g$ of $F_{2}$.

Proof. Gromov compactification is a coarse compactification, and isometric action on $X$ induces the continuous action on the boundary. If the induced action of $F_{2}$ on $\nu F_{2}$ has a fixed point, the induced action of $F_{2}$ on the Gromov boundary $\partial_{g} F_{2}$ also has a fixed point. However, one can easily see that for any point $z \in \partial_{g} F_{2}$, there exists an element $g$ of $F_{2}$ such that $g \cdot z \neq z$.

\section{$\S 3 . \quad$ Coarse fixed points}

Definition 3.1. Let $G$ be a finitely generated semigroup acting on $X$. We call a point $x$ of $X$ a coarse fixed point if its orbit $G \cdot x=\{g \cdot x: g \in G\} \subset X$ is a bounded set.

If $G$ is an infinite group and $x$ is a coarse fixed point, then the orbit of $x$ is not proper. In the following two propositions, the converse holds.

Proposition 3.2. Let $X$ be a metric space such that any bounded subset $D \subset X$ is a finite set. Suppose that $\mathbb{N}$ acts on $X$. Then a point of $X$ whose orbit is not proper is a coarse fixed point.

Proof. Let $x_{0}$ be a point in $X$ whose orbit is not proper. Then there exists a bounded set $D \subset X$ such that $\left\{n \in \mathbb{N}: n \cdot x_{0} \in D\right\}$ is an infinite set. Because $D$ is a finite set, there exist positive integers $m>n$ such that $m \cdot x_{0}=n \cdot x_{0}$. 
For any integer $l>m$, there exist integers $k>0$ and $r=0, \ldots, m-n-1$ satisfying $l-n=k(m-n)+r$. Thus, we have $l \cdot x_{0}=(n+r) \cdot x_{0}$. It follows that $\mathbb{N} \cdot x_{0} \subset\left\{x_{0}, 1 \cdot x_{0}, 2 \cdot x_{0}, \ldots,(m-1) \cdot x_{0}\right\}$.

Proposition 3.3. Let $X$ be a proper metric space. Suppose that $\mathbb{N}$ acts on $X$ by isometries. Then each point of $X$ whose orbit is not proper is a coarse fixed point.

Proof. Suppose that the orbit of $x_{0}$ is not proper. Then there exists a bounded set $D \subset X$ such that $\sharp\left\{n \in \mathbb{N}: n \cdot x_{0} \in D\right\}=\infty$. We can assume that $x_{0}$ lies in $D$. We notice that for any point $x$ of the orbit $\mathbb{N} \cdot x_{0}$, there exist $n(x) \in \mathbb{N}$ such that $n(x) \cdot x$ lies in $D$. We define a bounded subset $K \subset X$ by

$$
K=B(D, 1) \cap \mathbb{N} \cdot x_{0} .
$$

Here $B(D, 1)=\left\{x \in X:{ }^{\exists} y \in D, d(x, y)<1\right\}$ is the 1-neighborhood of $D$. Since $\bar{K}$ is a compact set, there exist $x_{1}, \ldots, x_{N} \in K$ such that

$$
\bar{K} \subset \bigcup_{i=1}^{N} B\left(x_{i}, 1\right) .
$$

For $0 \leq i \leq N$, we denote $T_{i}=n\left(x_{i}\right)$. The point $T_{i} \cdot x_{i}$ lies in $D$. We define a positive number $L$ by

$$
L=\max _{0 \leq i \leq N} \max _{0 \leq a \leq T_{i}} d\left(x_{0}, a \cdot x_{i}\right) .
$$

We define inductively a sequence $\left\{i_{k}\right\}_{k=0}^{\infty}$ consisting of integers in $\{1, \ldots, N\}$ and an increasing sequence $\left\{S_{j}\right\}_{j=0}^{\infty}$ as follows. Let $S_{0}=T_{0}$. Since $S_{0}$. $x_{0}=T_{0} \cdot x_{0} \in K$, there exists an integer $i_{0} \in\{1, \ldots, N\}$ such that $S_{0} \cdot x_{0} \in$ $B\left(x_{i_{0}}, 1\right)$. Assume that we have defined $i_{0}, \ldots, i_{n}$ and $S_{0}, \ldots, S_{n}$ such that, for $0 \leq j \leq n$ and $0 \leq a<T_{i_{j}}$, they satisfy

$$
\begin{gathered}
S_{j}=\sum_{k=0}^{j-1} T_{i_{k}}, \\
S_{j} \cdot x_{0} \in B\left(x_{i_{j}}, 1\right), \\
\left(a+S_{j}\right) \cdot x_{0} \in B\left(x_{0}, L+1\right) .
\end{gathered}
$$

Set $S_{n+1}=T_{i_{n}}+S_{n}$. Since $T_{i_{n}} \cdot x_{i_{n}} \in D$ and

$$
d\left(S_{n+1} \cdot x_{0}, T_{i_{n}} \cdot x_{i_{n}}\right)=d\left(S_{n} \cdot x_{0}, x_{i_{n}}\right)<1,
$$


we have $S_{n+1} \cdot x_{0} \in B(D, 1)$, and thus $S_{n+1} \cdot x_{0}$ lies in $K$. Hence, there exists an integer $i_{n+1}$ such that $S_{n+1} \cdot x_{0}$ lies in $B\left(x_{i_{n+1}}, 1\right)$. For $0 \leq a<T_{i_{n+1}}$, we have $d\left(x_{0}, a \cdot x_{i_{n+1}}\right) \leq L$ and

$$
d\left(\left(a+S_{n+1}\right) \cdot x_{0}, a \cdot x_{i_{n+1}}\right)=d\left(S_{n+1} \cdot x_{0}, x_{i_{n+1}}\right)<1
$$

It follows that $\left(a+S_{n+1}\right) \cdot x_{0}$ lies in $B\left(x_{0}, L+1\right)$. This shows that for any integer $l>0, l \cdot x_{0}$ lies in $B\left(x_{0}, L+1\right)$, and thus $x_{0}$ is a coarse fixed point.

The hypothesis that $X$ is a proper space is necessary (see [3, Exercise 5.2.26]).

REMARK 3.4. Under the assumption of Proposition 3.3, if $x_{0}$ is a coarse fixed point, then any point $x$ of $X$ is a coarse fixed point.

If the orbit is not coarse, there are two possibilities; namely, the orbit is either nonproper or nonbornologous. However, if the action is an isometry, any orbit is bornologous.

LEMMA 3.5. Let $X$ be a proper metric space with an isometric action of $\mathbb{N}$. Then the action is a coarse action, and any orbit is bornologous.

Proof. An isometric action is a coarse action. For any given point $x$ of $X$, put $L=d(1 \cdot x, x)$. Then we have $d((i+1) \cdot x, i \cdot x)=L$ for all integers $i>0$. Hence, for any integers $m \geq n>0$, we have

$$
d\left(\Phi_{x}(m), \Phi_{x}(n)\right)=d(m \cdot x, n \cdot x) \leq \sum_{i=n}^{m-1} d((i+1) \cdot x, i \cdot x)=L|m-n| .
$$

Thus, $\Phi_{x}$ is a bornologous map.

Proof of Corollary 1.7. An isometric action of $\mathbb{N}$ on $X$ is defined by $(n, x) \mapsto f^{n}(x)$. If $f$ has no coarse fixed point, then by Proposition 3.3 and Lemma 3.5, any orbit of this action is coarse. Thus, Theorem 1.3 implies that $\bar{f}$ has a fixed point.

\section{$\S 4$. Another example of coarse action}

There exists a coarse map which has no coarse fixed points on the coarse space or fixed points on its boundary. 


\section{Adding machine}

Let $T_{2}=\left(V_{2}, E_{2}\right)$ be a binary tree with the set of vertices $V_{2}$ and the set of edges $E_{2}$. We identify $V_{2}$ with the following sets:

$$
V_{2} \cong\{*\} \sqcup \bigsqcup_{n \geq 1}\{0,1\}^{n} .
$$

Here $*$ denotes the base point:

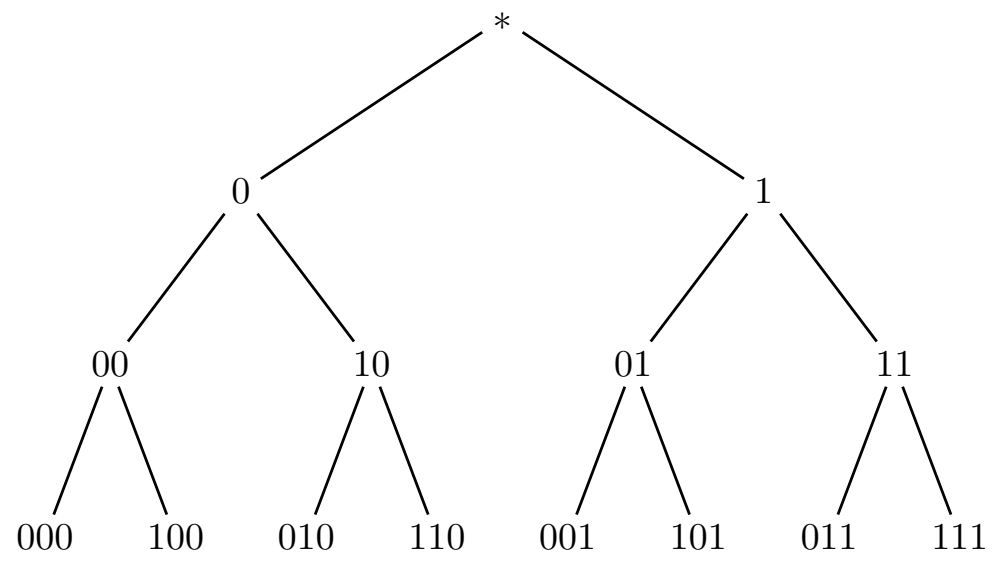

* and 0 (resp., 1) can be joined by the edge $e_{*, 0}$ (resp., $e_{*, 1}$ ). Let $x=$ $\left(i_{n-1}, \ldots, i_{0}\right)$ and $y=\left(j_{n}, \ldots, j_{0}\right)$ be two vertices. Both $x$ and $y$ can be joined by the edge $e_{x, y}$ if and only if $i_{0}=j_{0}, \ldots, i_{n-1}=j_{n-1} \cdot T_{2}$ has the usual metric and is a geodesic space. $V_{2}$ has the induced metric from $T_{2}$.

We construct a coarse action of $\mathbb{N}$ on $V_{2}$. Let $x$ be a vertex. If $x=$ $\left(i_{n}, \ldots, i_{0}\right) \neq(1, \ldots, 1)$, we define $1 \cdot x=\left(j_{n-1}, \ldots, j_{0}\right)$ with

$$
\sum_{k=0}^{n-1} j_{k} 2^{k}=\sum_{k=0}^{n-1} i_{k} 2^{k}+1 .
$$

If $x=(\overbrace{1, \ldots, 1}^{n})$, we define $1 \cdot x=(\overbrace{1,0, \ldots, 0}^{n+1})$. Finally, we define $1 \cdot *=(0)$.

The Gromov product of $x=\left(i_{n-1}, \ldots, i_{0}\right)$ and $y=\left(j_{m-1}, \ldots, j_{0}\right)$ with a base point $*$ is defined to be

$$
(x \mid y)=\frac{1}{2}\{d(x, *)+d(y, *)-d(x, y)\} .
$$

We notice that $(x \mid y)=r$ if and only if $i_{0}=j_{0}, \ldots, i_{r-1}=j_{r-1}$ and $i_{r} \neq j_{r}$. 
LEMMA 4.1. $1 \cdot: V_{2} \rightarrow V_{2}$ is a coarse map.

Proof. Let $x=\left(i_{n-1}, \ldots, i_{0}\right)$ and $y=\left(j_{m-1}, \ldots, j_{0}\right)$ be two vertices. Set $(x \mid y)=r$. Then $i_{0}=j_{0}, \ldots, i_{r-1}=j_{r-1}$. It is easy to see that $(1 \cdot x \mid 1 \cdot y) \geq r$. Hence, we have

$$
\begin{aligned}
d(1 \cdot x, 1 \cdot y) & =d(1 \cdot x, *)+d(1 \cdot y, *)-2(1 \cdot x \mid 1 \cdot y) \\
& \leq(n+1)+(m+1)-2 r \\
& =d(x, y)+2
\end{aligned}
$$

Thus, $x \mapsto 1 \cdot x$ is a large-scale Lipschitz map. Since the map $1 \cdot$ is injective and every bounded set of $V_{2}$ is a finite set, the map $1 \cdot$ is proper.

This action can be extended to a continuous action on the Gromov boundary $\partial_{g} V_{2}$ of $V_{2}$. Here we can identify $\partial_{g} V_{2}$ as the Cantor set $\{0,1\}^{\mathbb{N}}$ with a metric $d$ defined to be

$$
d(x, y)=2^{-(x \mid y)} .
$$

Here $x=\left(i_{k}\right)_{k=0}^{\infty}$, and $y=\left(j_{k}\right)_{k=0}^{\infty}$.

Proposition 4.2. The action of $\mathbb{N}$ on $\partial_{g} V_{2}$ is minimal. Namely, for any point $x$ of $\partial_{g} V_{2}$, its orbit $\mathbb{N} \cdot x$ is dense in $\partial_{g} V_{2}$.

Proof. Let $x \in \partial_{g} V_{2}$ be given. For any point $y$ of $\partial_{g} V_{2}$ and $\epsilon>0$, we will show that there exists an integer $n$ such that $d(n \cdot x, y)<\epsilon$. Set $N>$ $-\log \epsilon / \log 2$, and set $x=\left(i_{k}\right)_{k=0}^{\infty}$. We choose $a=2^{N+1}-\sum_{k=0}^{N} i_{k} 2^{k} ;$ then we have

$$
a \cdot x=(\ldots, \overbrace{0, \ldots, 0}^{N+1}) .
$$

Set $y=\left(j_{k}\right)_{k=0}^{\infty}$, and set $b=\sum_{k=0}^{N} j_{k} 2^{k}$. Then we have

$$
(a+b) \cdot x=\left(\ldots, j_{N}, \ldots, j_{0}\right)
$$

It follows that $d((a+b) \cdot x, y)<2^{-N}<\epsilon$.

In particular, the action of $\mathbb{N}$ on $\partial_{g} V_{2}$ has no fixed point. Moreover, the action has no coarse fixed point in $V_{2}$. Thus, by Proposition 3.2 and Theorem 1.3, the orbit of $x \in V_{2}$ can never be bornologous. 


\section{Appendix}

\section{Metric of the cone and its compactification}

Let $M$ be a compact path metric space. The cone $C M$ on $M$ is the quotient space $M \times[0, \infty) / \sim$, where $(x, t) \sim\left(x^{\prime}, t^{\prime}\right)$ if and only if either $x=x^{\prime}$ and $t=t^{\prime}$ or $t=t^{\prime}=0$. According to Roe [4, Section 3.6], we construct a metric on $C M$. Let $\lambda:[0, \infty) \rightarrow[0, \infty)$ be a continuous function with $\lambda(t)=0$ if and only if $t=0$. If $\gamma$ is a path in $C M$, we define its $\lambda$-length, $l_{\lambda}(\gamma)$, to be

$$
\sup \left\{\sum_{j=0}^{n-1}\left(\left|t_{j}-t_{j+1}\right|+\max \left\{\lambda\left(t_{j}\right), \lambda\left(t_{j+1}\right)\right\} d\left(x_{j}, x_{j+1}\right)\right)\right\},
$$

where the supremum is taken over all finite sequences $\left(x_{j}, t_{j}\right)_{j=0}^{n}$ of points on the path $\gamma$ (with $\left(x_{0}, t_{0}\right)$ and $\left(x_{n}, t_{n}\right)$ being two endpoints). We define a metric $d_{\lambda}$ on $C M$ by $d_{\lambda}\left((x, t),\left(x^{\prime}, t^{\prime}\right)\right)=\inf l_{\lambda}(\gamma)$, where the infimum is taken over all paths joining $(x, t)$ and $\left(x^{\prime}, t^{\prime}\right)$. The metric $d_{\lambda}$ is compatible with the topology of $C M$, and it becomes a proper metric space (see $[4$, Proposition 3.47]).

Proposition A.1. Suppose that $\lambda$ is an increasing, unbounded function; then the compactification $\overline{C M}=M \times[0,1] / \sim$ is a coarse compactification.

Proof. Let $E \subset C M \times C M$ be a controlled set, and let $r_{E}$ be a positive number such that

$$
\sup \left\{d_{\lambda}(\mathbf{x}, \mathbf{y}):(\mathbf{x}, \mathbf{y}) \in E\right\}<r_{E} .
$$

Let $\left\{\left(\mathbf{x}_{n}, \mathbf{y}_{n}\right)\right\} \subset E$ be a sequence such that $\mathbf{x}_{\mathbf{n}}$ and $\mathbf{y}_{\mathbf{n}}$ tend to $x$ and $y$ in $\partial(C M) \cong M$, respectively. We denote $\mathbf{x}_{n}=\left(x_{n}, t_{n}\right)$ and $\mathbf{y}_{n}=\left(y_{n}, s_{n}\right)$. It is enough to show that $x=y$ (see [5, Theorem 2.27]). Suppose that $x \neq y$. Since $x_{n}$ and $y_{n}$ converge to $x$ and $y$, respectively, for any $\epsilon>0$ there exists $N_{1}>0$ such that, for all $n>N_{1}, x_{n}$ and $y_{n}$ satisfy $d\left(x_{n}, y_{n}\right)>d(x, y)-\epsilon$. Because $\lambda$ is an increasing, unbounded function, there exists $N_{2}>0$ such that, for all $t>N_{2}$, we have

$$
\lambda(t)>\frac{r_{E}}{d(x, y)-\epsilon} .
$$

Since $\mathbf{x}_{\mathbf{n}}$ tends to infinity, there exists $N_{3}>0$ such that, for all $n>N_{3}$, the second component $t_{n}$ of $\mathbf{x}_{\mathbf{n}}$ satisfies $t_{n}>N_{2}$. Thus, if we choose an integer $n$ satisfying $n>\max \left\{N_{1}, N_{3}\right\}$, then we have $d_{\lambda}\left(\mathbf{x}_{n}, \mathbf{y}_{n}\right)>r_{E}$; this contradicts the definition of $r_{E}$. 
Acknowledgments. We thank the participants in the coarse geometry seminar at Kyoto University - S. Honda, T. Kato, T. Kondo, and M. Tsukamoto — for several discussions and useful comments.

\section{REFERENCES}

[1] A. N. Dranishnikov, J. Keesling, and V. V. Uspenskij, On the Higson corona of uniformly contractible spaces, Topology 37 (1998), 791-803.

[2] É. Ghys and P. de la Harpe, Sur les groupes hyperboliques d'après Mikhael Gromov, Prog. Math. 83, Birkhäuser, Boston, 1990.

[3] A. Navas, Groups of Circle Diffeomorphisms, Chicago Lectures Math., University of Chicago Press, Chicago, 2011.

[4] J. Roe, Coarse cohomology and index theory on complete Riemannian manifolds, Mem. Amer. Math. Soc. 104 (1993), no. 497.

[5] - Lectures on Coarse Geometry, University Lecture Ser. 31, Am. Math. Soc., Providence, 2003.

Department of Mathematics

Kyoto University

Kyoto 606-8502

Japan

tomo@math.kyoto-u.ac.jp 